\title{
A virtual dissection based registration to model patient-specific respiratory motion
}

\author{
John Jones, Member, IEEE, Emma Lewis, Matthew Guy, and Kevin Wells
}

\begin{abstract}
This work is directed at reducing patient induced blurring in SPECT imaging due to breathing motion. As image resolution improves this breathing motion is becoming increasingly significant.

Method: The NCAT phantom and an associated medical image processing package (RMDP) are used to obtain a breathing cycle of images (both CT and corresponding SPECT) and a full organ segmentation. A process termed 'virtual dissection' is undertaken which sees individual organs extracted from the main images and independently registered (ICP). These individual registrations are reconciled, combined and used to obtain improved final images.

Results: The results of the objective validation techniques are presented together with a comparison of processed and unprocessed images.

Conclusion: Within the scope of the synthetic data used and for organs for which the assumption of near-rigid motion holds well the technique works. In the case of the ribs and lungs further development is needed
\end{abstract}

Index Terms-registration, respiratory, breathing, blurring, Iterated Closest Points, ICP, affine, rigid, NCAT, XCAT, Nuclear Medicine Imaging, SPECT, PET.

\section{INTRODUCTION}

$\mathbf{N}$ UCLEAR Medicine (NM) Images show the spatial variations in concentration of an injected or inhaled radiopharmaceutical. Such variations can indicate the presence of disease well before being anatomically present, which explains its continuing popularity despite the drawbacks of slow acquisition and noisy, blurred images. Patient induced blurring due to breathing motion is becoming increasingly significant as instrument resolution improves.

Registration of 3D Medical Images can be categorized in a number of ways. One such categorization is the type of transformation involved which may be rigid, affine or nonaffine. Arun et al have described a closed form solution of the rigid 3D problem which Besl and McKay used as part of their practical algorithm for multidimensional rigid registration expressed in a variety of data representations which later became known as Iterated Closest Points (ICP) [1], [2], [3]. $\mathrm{Du}$ et al modified the algorithm from rigid (6 degrees of freedom) to affine (12 dof) [4]. Another way of categorizing registrations is by whether they use voxel intensity values directly or employ segmentation to construct a model of the shape of related tissue and process that, as is done for ICP. One intensity-based registration tool is IRTK, which uses cubic

J. Jones, E. Lewis and K. Wells are with the Centre for Vision, Speech and Signal Processing, University of Surrey, Guildford, Surrey GU2 7XH, UK. e-mail: j.jones e.lewis k.wells @ surrey.ac.uk

M. Guy is with the Department of Physics, University of Surrey, Guildford, Surrey GU2 7XH, UK. e-mail: Matthew.Guy@nhs.net

Manuscript received November 6, 2009. splines to model smooth deformations[5], [6]. In this method there is a 3D grid of control points and the user may choose from a variety of similarity measures and a variety of methods of optimization.

In the project described here we start from a breathing cycle image dataset acquired from dynamic X-ray Computed Tomography (CT) and corresponding NM images obtained from the NCAT phantom and an associated medical image processing package (RMDP). A novel procedure, termed "virtual dissection", is undertaken where each organ is individually registered and each registration is expressed as a motion field. This is part of a wider project at the University of Surrey which aims to address the problem of breathing motion by combining the construction of patient specific models of internal abdominal/thoracic motion using low dose dynamic CT data with dynamic stereo tracking of the patients skin surface and a novel particle filter approach to link these components[7], [8], [9]. Three techniques are compared. ICP (6 dof) is a Matlab implementation based on a $\mathrm{C}++$ implementation by Bergstrom and Shechter [10]. ICP(12 dof $)$ is a Matlab implementation based on the Du et al. paper [4]. IRTK is an intensity based non-rigid registration package [5], [6] based on hierarchical cubic B-spline grids.

\section{MethoD}

\section{A. Process Flowchart}

Figure II-A shows the flowchart of the ICP registration process. The process as applied to single organs for validation purposes is indicated to cover steps 1 to 4 . Step 5 is used only during the 'virtual dissection' process.

1 Obtain Segmented Organ For each major organ the reference frame is paired with each of the other frames of the breathing cycle. Given a breathing cycle of eight frames there are seven pairs for comparison.

2 Convert to point-cloud surfaces The segmented organs, supplied by NCAT as 3-D images, are converted to surfaces using the commonly used morphological operations. These are in turn converted to point cloud representation.

3 Register surfaces (ICP) The two surfaces are registered using a Matlab implementation of the ICP algorithm developed for this project. The result is a description of the transformation from reference surface to source surface in the form of an affine transformation matrix plus a translation vector.

4 Express registration as a motion field The concise description of the registration is reversed and evaluated at each voxel of the organ in the reference frame to derive a motion field from the data image to the reference image. 


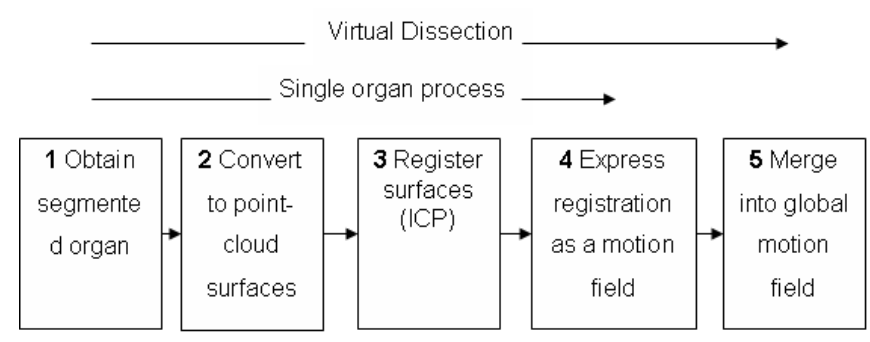

Fig. 1. Process flowchart covering the ICP registrations. The numbered boxes are expanded in the text of II-A.

5 Merge results into global motion field A single global motion field is maintained for each comparison frame. Where, in rare cases, the same voxel is specified for more than one organ the vector average of all specifications is calculated. This step is required only during virtual dissection.

\section{B. Validation}

In addition to visualisation of the lesions two methods of evaluating the results were used.

1) Consistently labelled fraction measure: Considering the reference image in its coordinate system and the source image registered into the same coordinate system, in the case of a perfect registration the two images overlap perfectly and all the image voxels are consistently labelled as being in the combined image. In practise, the registration is not perfect and there are some voxels of the combined images that are only in one of the separate images. These are said to be inconsistently labelled. If the image components are $\mathrm{A}$ and $B$ and $\#()$ is an operator returning the number of voxels of the operand, then the measure selected here is the fraction of voxels consistently labelled or $\mathrm{C}$ where $C=\frac{\#(A \cap B)}{\#(A \cup B)}[11]$. This measure is used for the results of the serial individual organ registrations (Section III).

2) Comparison with ground-truth: In this context the ground-truth is a vector displacement map (VDM) output by NCAT. Given the coordinates of some tissue in frame 1 the VDM may by used to derive the coordinates of the same tissue in frame 2. This process may be repeated to derive the motion throughout a breathing cycle. Considering the coordinates of the centre of a lesion in frame 1, the VDM is used to derive its coordinates in frame 2 and the motion field resulting from the registrations of frame 2 organs may be used to give the centre of the lesion after registration. Ideally these will match the frame 1 coordinates but in practise there is a difference. If we consider the complete set of registered lesion centres, the diameter of the set is used as a measure of the registrations effectiveness and would ideally be zero.

\section{Creating the simulated NM and CT data}

The project has used simulated CT and NM data generated by NCAT[12] and The Radionuclide Multimodality Dosimetry Package (RMDP) [13]. The NM data has a number of lesions placed in it. The data comprises breathing cycles of eight frames.

\section{Serial Organ Registration}

Serial Organ Registration is a procedure carried out when validating the virtual dissection. Serial organ registration treats each major organ completely separately. It is usually run in a sequence for each major organ. For each major organ the reference frame is compared with each of the other frames of the breathing cycle. Given a breathing cycle of eight frames there are seven comparisons for each of three types of registration: ICP (twelve dof), ICP(six dof) and IRTK. The pairs of images are processed as described in II-A (with the omission of the 5th step which applies only to virtual dissection proper). The motion field is applied to the source segment image and the results are then analysed using the "fraction of consistently labelled voxels" as described above in II-B1.

\section{E. Virtual dissection}

Virtual dissection produces a registered image of the whole torso. The basic routines are identical or very similar to those of serial organ registration but they are composed differently. Organs are divided into two groups: simple organs, and complex organs.

The simple organs (heart, liver, spleen, gall-bladder, stomach and intestines) are handled sequentially as independent ICP registration tasks. The complex organs (lungs, ribs and skin) are then processed in that particular order. The final task is to impute data for any remaining tissue.

The modus operandi is to start with a complete image and to extract each organ in turn whilst maintaining an image of the union of all removed tissue and an image of residual voxels after each organ removal. In addition, a single global motion field per comparison frame is gradually built up.

1) The simple organs: Each simple organ in turn is processed as described in II-A. However, unlike in serial organ registration, all organ registrations go into the global motion field. This common or mosaic motion field is held as four 3D images. Three of the images hold the $\mathrm{x}, \mathrm{y}$, and $\mathrm{z}$ components of a relative motion in millimetres. Whenever a voxel is included in an organ, the contribution is accumulated in the voxels of these images and the voxels of the fourth image hold the number of contributors so far for the voxel in question. This number of contributors is used later to average the $\mathrm{x}, \mathrm{y}$ and $\mathrm{z}$ components.

As each simple organ is processed its volume is marked in a "UnionOfOrgans" image with the sequence number of the organ being processed. This image forms part of the interface with the Particle Filter stage of the larger University of Surrey project and the sequence number identifies the organ within the matrix which forms the main part of the interface.

The further "residual data image" that is maintained during this sequence is "Remains". As its name suggests, the image originates as a copy of the source frame and as each organ is processed the voxels of the organ are set to the background value. The reason for maintaining such an image will become clear when describing the treatment of the ribs. 
2) The lungs: Once the aforementioned sequence of simple organs has been processed the lungs are considered.

The lungs are an area where rigid motion is least likely to hold. Initial investigation simply divides them horizontally into a selected number of equal height slabs. Two, three and four divisions are made resulting in four, six and eight lung components. Each of the components is handled independently in the same manner as the simple organs. (See II-A.)

The lungs are processed before the ribs because some of the rib-related processing of the "Remains" image would be incorrect if lung tissue remained.

3) The ribs: After the lungs have been removed the ribs are processed. NCAT delivers the ribs as a single organ. For comparison purposes the ribs are processed as a single organ in the serial organ registration and, in virtual dissection, each half rib is treated independently.

One feature of processing unique to the ribs is that, after the individual ribs have been labelled, tissue adjacent to a rib is tied to that particular rib. Since the lungs have been removed before this stage this association affects only the outer surface of the ribs and intercostal tissue. When the affine matrix and translation which describe the registration are converted to a motion field it is extended both over the ribs and the extra associated tissue. Thus intercostal tissue is deemed to move with the corresponding rib.

4) The skin: The skin is handled in a manner similar to the lung. The outer skin surface is constructed using standard morphological operations. Using the centre of the image as the centre of a circle the surface is divided into a number of sectors (in this instance, six) around the transverse axis. Then transverse divisions are made (in this instance, four).

The end result is a number of plates (24) which, when converted to point-cloud format are individually registered to the equivalently constructed plates of the reference image.

5) Interpolation: The penultimate step in virtual dissection is to consider those voxels of the reference coordinates which do not have a motion vector indicating the source coordinates of the alignment. The process is termed interpolation.

The approach taken is a simple, non-isotropic, inverse distance weighting. From each point requiring interpolation, and for each of the 6 coordinate directions, a search is made at increasing distance from the point being interpolated. The search is for the first voxel that has a motion vector assigned by the processing of the organs described above. Thus there may be from zero to six motion vectors, termed contributors, to the inverse distance weighting procedure. Interpolation is skipped if there is no pair of contributors that bracket the point being interpolated. Since by this stage there are plates of skin (possibly with gaps) surrounding the partly registered torso, it is almost invariably true, from empirical observation, that at least one and usually three bracketing pairs exist. Thus interpolation completes the motion field.

6) Final steps: Finally, with a complete motion field, the source image is re-sliced using sinc interpolation to form a final registered image. In the case of NM imaging all resliced images and the reference image are summed to form the final output image.
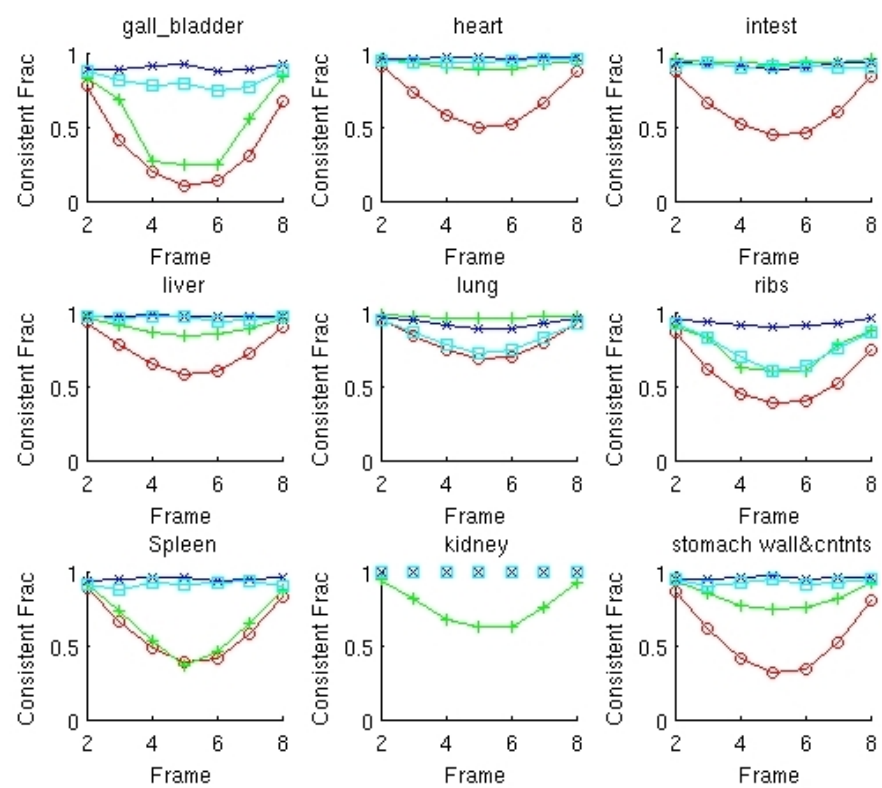

Fig. 2. Individual organs pre and post registration consistently labelled fraction statistic. Red: No registration, Blue: ICP(12 dof), Cyan: ICP(6 dof, Green: IRTK. The reference or model image is frame 1. The results are from NCAT and are from a single respiratory cycle comprised of 8 frames.

\section{RESULTS}

Figure 2 shows the results of running the organs serially. The values plotted are the consistently labelled fraction statistic. For all organs except the lungs and intestines, ICP with the twelve dof option is an improvement on six dof and on IRTK for the consistent fraction measure, although for the heart the improvement is marginal.

The range of the consistent fraction for the gall bladder is large (approx $0.1-0.9$ ) because it is a small organ and the size of its translation almost separates the source and reference images in the comparison. Twelve dof ICP shows better alignment than six dof ICP. For the lungs, which in this context are treated as a single shape, there is a clear improvement between six degrees of freedom and twelve, with six dof being little better than no registration. IRTK gives a slightly better result than 12 dof. The ribs are treated as a single shape. There is again a clear improvement comparing six dof to twelve. This time however IRTK produces similar results to ICP with six dof. There is little difference between six dof and twelve dof, for the spleen, indicating less distortion due to breathing. IRTK is no improvement on no registration. In the version of NCAT used, the kidneys are not moved during the breathing cycle, hence the consistent fraction has a value of one for ICP and no registration. The apparent movement in the IRTK is a result of the first step intrinsic to IRTK being to find the optimal affine transformation over the whole torso. This results in kidney motion which is not fully restored by the non-rigid stage of IRTK.

Figure 3 shows the result of dividing the lungs into separate components for independent registrations. A number of transverse slices are made between the superior and inferior extremes of the lungs. One slice results in four lung components, two slices in six and three slices in eight components. The 

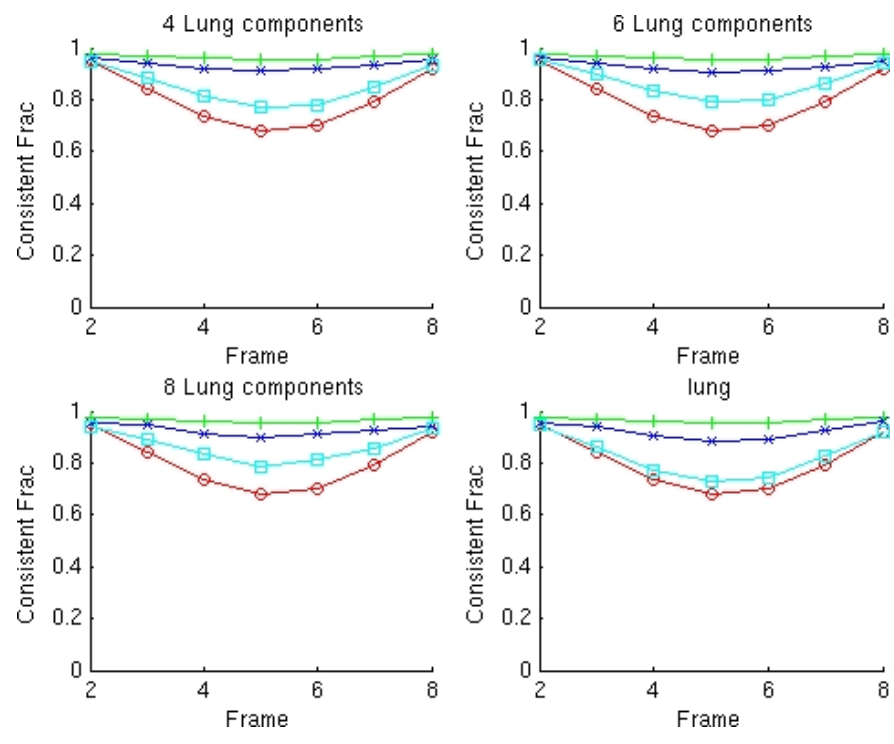

Fig. 3. Comparison of different treatments of the Lungs (consistently labelled fraction statistic); plotted using the same scheme as the previous figure 2 .
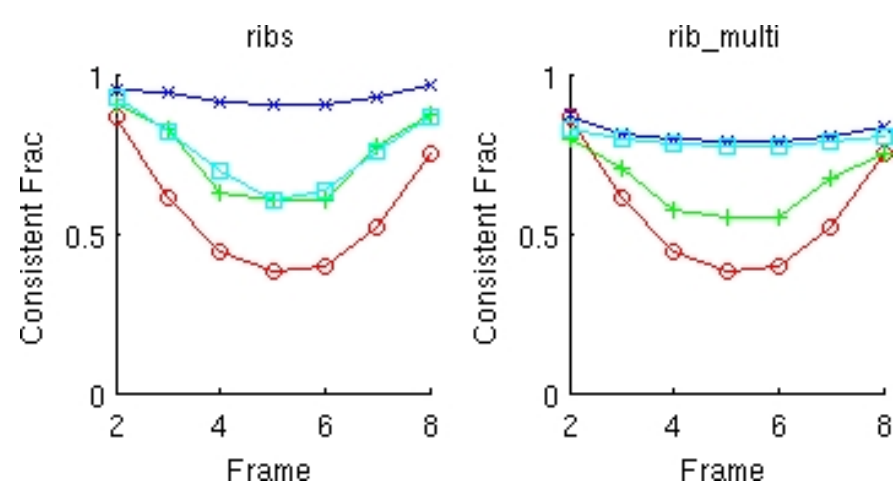

Fig. 4. Comparison of entire rib-cage against multiple half-ribs. It shows that extending from 6 to 12 dof is superior to treating ribs separately; plotted using the same scheme as 2

\begin{tabular}{|c|c|c|c|c|c|c|c|}
\hline \multirow[b]{2}{*}{$\begin{array}{l}\text { Lesion } \\
\text { Number }\end{array}$} & \multirow[b]{2}{*}{$\begin{array}{c}\text { Initial } \\
\text { Diameter* }\end{array}$} & \multicolumn{2}{|c|}{ ICP Affine (12 dof) } & \multicolumn{2}{|c|}{ ICP Rigid (6 dof) } & \multicolumn{2}{|c|}{ IRTK (B-Splines) } \\
\hline & & $\begin{array}{c}\text { Final } \\
\text { Diameter }\end{array}$ & $\begin{array}{l}\text { \% Separation } \\
\text { Remaining }\end{array}$ & $\begin{array}{c}\text { Final } \\
\text { Diameter }\end{array}$ & $\begin{array}{c}\text { \% Separation } \\
\text { Remaining }\end{array}$ & $\begin{array}{c}\text { Final } \\
\text { Diameter }\end{array}$ & $\begin{array}{c}\text { \% Separatior } \\
\text { Remaining }\end{array}$ \\
\hline 1 & 7.04 & 0.66 & 9.39 & 1.22 & 17.33 & 2.19 & 31.04 \\
\hline 2 & 6.46 & 1.76 & 27.26 & 2.4 & 37.09 & 2.84 & 43.89 \\
\hline 3 & 7.04 & 0.59 & 8.33 & 1.2 & 17.09 & 9.78 & 138.91 \\
\hline 4 & 4.78 & 0.92 & 19.33 & 1.27 & 26.49 & 1.36 & 28.46 \\
\hline 5 & 2.65 & 0.73 & 27.6 & 2.01 & 75.71 & 1.9 & 71.67 \\
\hline
\end{tabular}

Fig. 5. Post registration spread of the lesion centres. * pre registration diameter of lesion centres, **post registration diameter of lesion centres, *** final diameter as percentage of initial diameter

results indicate that twelve dof without subdivision is superior to six dof with or without subdivision. The extension to twelve degrees of freedom renders lung subdivision superfluous. A comparison of the rib-cage as a single shape and treating each half-rib as an independent shape is shown in Figure 4. The technique of sub-dividing the ribs offers no advantage over simply extending from six dof to twelve dof whilst treating the ribs as a single shape.

Figure 5 shows the results of examining the centres of 5

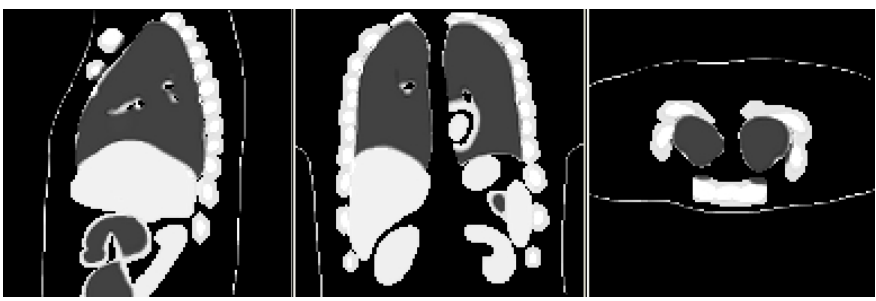

Fig. 6. Complete virtual dissection immediately prior to interpolation.

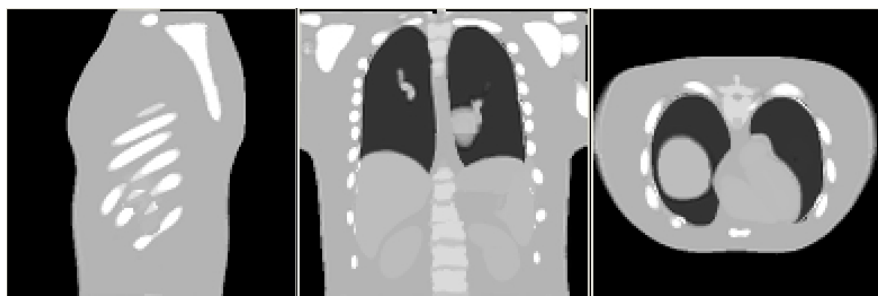

Fig. 7. Virtual dissection after completion.

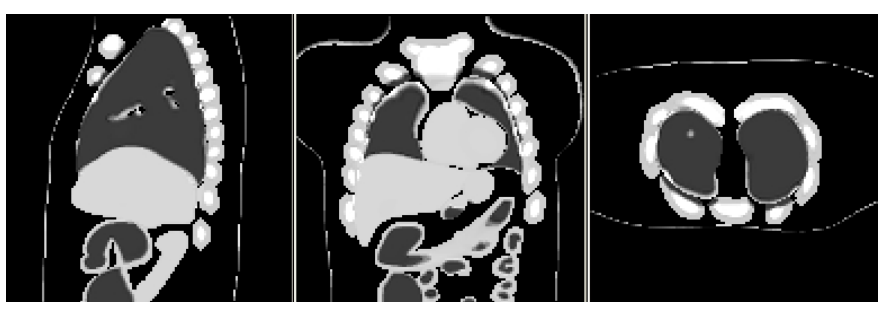

Fig. 8. Worst view of the image prior to interpolation. It shows artefacts near the top of the lungs

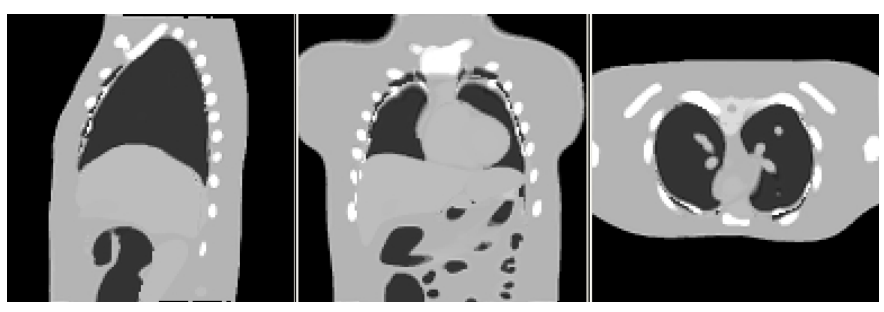

Fig. 9. Worst view of the image of completed virtual dissection. Again showing artifacts near the top of the lungs.

lesions placed in the NM images using the RMDP package. The lesions are not present in the CT images so can have no influence on the registrations themselves. The "diameter" of the set of lesion centres is the diameter of the smallest sphere which can contain them. The ideal final diameter is zero which occurs when all registered lesion centres coincide. These diameters are not the lesion diameters. The results in table 4-1 show a clear improvement for ICP over IRTK and ICP with twelve dof over ICP with six dof.

Both the CT and NM images may be viewed to visually assess the quality of the registration. CT images are shown here because important detail is not discernable in the NM images. The images shown are from the registration over the largest possible registration (full-exhalation back to fullinhalation or frame 5 back to frame 1). Figure 6 shows 


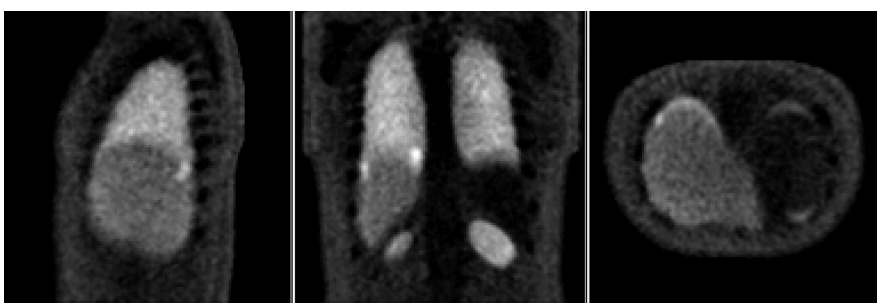

Fig. 10. Unregistered NM images summed.

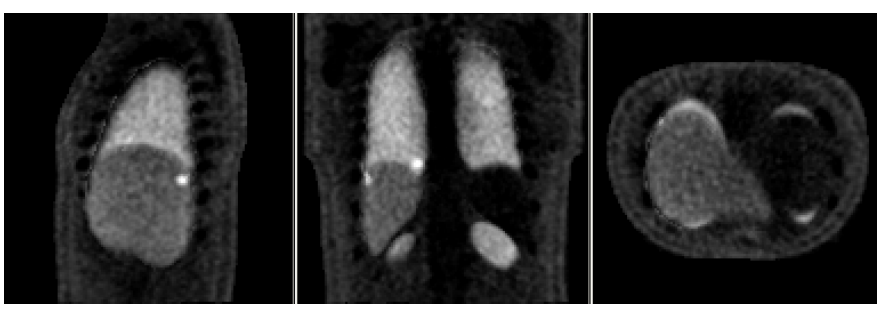

Fig. 11. NM images registered with ICP six dof and summed.

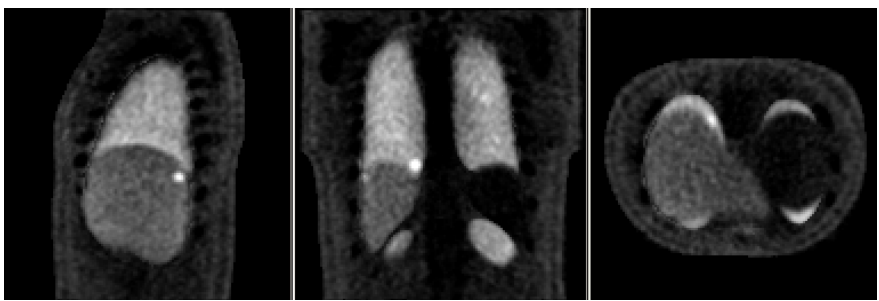

Fig. 12. NM images registered with ICP twelve dof and summed.

images of a virtual dissection after the major organs have been processed but before the final inrterpolation has been done. It shows the ribs with the intercostal tissue attached. Figure 7 shows the situation once the interpolation has been completed. The images in Figure 6 and Figure 7 were chosen to represent the better end of the spectrum of results obtained. Figure 8 and Figure 9 were included to show the worst situation and show artefacts particularly where the front of the lungs meet the chest.

The final three figures indicate the effect of the virtual dissection procedure on the NM images produced. Figure 10 shows the effect of taking the unregistered NM images and simply summing them together. It is the best approximation available to what would be seen if a single ungated image were acquired. The elongation of the lesions is visible compared with the next two images. Figure 11 is the sum of all the NM images output by the ICP with six dof registrations and reflects the reduced blurring of the lesions. Figure 12 corresponds to Figure 11 but for ICP with twelve dof .

Finally Figure 13 shows a section of the motion field resulting from the registration between frames 1 and 5 . It is centred on voxel $(28,65,80)$ which is situated in the lung above the liver and adjacent to the ribs. It shows a small parallelopiped of voxel centres (small circles) and the vectors to the points in the source image which are to be interpolated to obtain the resliced voxels. The ribs and intercostal tissue

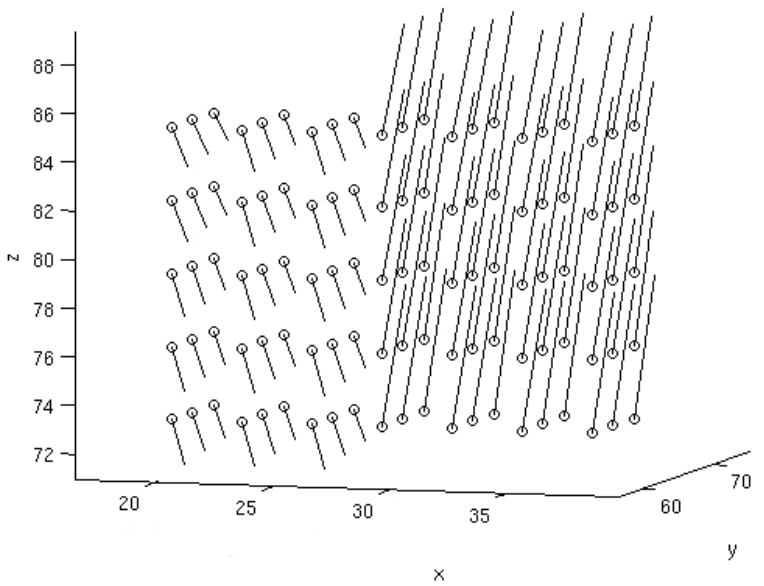

Fig. 13. A section of motion field resulting from registering frame 5 to frame 1. x: Lateral; y:Anterior-Posterior z:Inferior-Superior. The centre of the 3D grid is positioned in the lung above the liver and adjacent to the ribs

on the left of the figure are seen to move upwards (during inspiration) and immediately adjacent in the lung to the right of the figure the lung tissue can be seen moving sharply downwards as the lung expands.

This is a feature that was intended to be present in the mosaic motion fields produced by the virtual dissection and which is not present in the motion fields resulting from using cubic splines as in IRTK whose smooth output minimizes such features.

\section{CONCLUSION}

The results presented show that for organs such as liver, gall-bladder, spleen and kidneys, for which the assumption of near rigid motion holds well, ICP gives better results than IRTK. However, for the lungs IRTK still has the advantage. Extending the ICP implementation from rigid (six dof) to affine (twelve dof) made a larger improvement than dividing the lungs, albeit in a very simple manner, or treating each half-rib independently. Unfortunately the registered CT images show artefacts at the upper rib/lung boundary. The work completed thus far has provided a registration for use in breathing motion correction. Applying this technique to real data is a key item for future work. The approach taken in this work was an alternative to other non-affine techniques and is novel. These other techniques generally expend considerable computational effort to constrain their solutions to be "reasonable or likely" rather than near optimal but physically "unlikely or impossible". It is hoped that such constraints can be avoided or reduced in our approach and that our process of measuring and assembling the observed motions would avoid such gross errors as tearing or one section of tissue passing through another. However, at the "interpolation" stage of the virtual dissection anomalies would be introduced if it were not for the tagging of intercostal tissue to the closest rib. During interpolation in the intercostal region it is possible to include, as a contributor, a voxel within the lung. In that 
situation the sharply downward moving lung tissue may overinfluence the sharply upward moving intercostal tissue. The solution currently adopted is to associate intercostal tissue with its nearest rib. When, later, interpolation is executed, contributors do not influence voxels on the other side of the

rib and intercostal barrier. Initial simulation results suggest this works well.

\section{ACKNOWLEDGMENT}

The authors would like to thank the United Kingdom EPSRC for supporting this work.

\section{REFERENCES}

[1] K. Arun, T. Huang, and S. Blostein, "Least square fitting of two 3-d point sets," IEEE Transactions on Pattern Analysis and Machine Intelligence, vol. 9, no. 5, pp. 698-700, 1987.

[2] B. K. P. Horn, "Closed-form solution of absolute orientation using unit quaternions," Journal of the Optical Society of America a-Optics Image Science and Vision, vol. 4, no. 4, pp. 629-642, 1987, times Cited: 759.

[3] P. J. Besl and N. D. McKay, "A method for registration of 3-d shapes," IEEE Transactions on Pattern Analysis and Machine Intelligence, vol. 14, no. 2, pp. 239-256, 1992, times Cited: 2121.

[4] S. Y. Du, N. N. Zheng, G. F. Meng, and Z. J. Yuan, "Affine registration of point sets using icp and ica," IEEE Signal Processing Letters, vol. 15, pp. 689-692, 2008, times Cited: 0.

[5] D. Rueckert, L. I. Sonoda, C. Hayes, D. L. G. Hill, M. O. Leach, and D. J. Hawkes, "Nonrigid registration using free-form deformations: Application to breast mr images," IEEE Transactions on Medical Imaging, vol. 18 , no. 8, pp. 712-721, 1999.

[6] J. Schnabel, "A generic framework for non-rigid registration based on non-uniform multi-level free-form deformations." in Fourth Int. Conf. on Medical Image Computing and Computer- Assisted Intervention (MICCAI '01), Utrecht, NL, 2001, pp. 573-581.

[7] K. Wells, B. Goswami, A. Rahni, J. Jones, and E. L. M. Alnowami, "A flexible approach to motion correction in nuclear medicine," in IEEE Medical Imaging Conf. Orlando, Florida, USA: IEEE Medical Imaging Conf, 2009.

[8] A. Rahni, E. Lewis, M. Guy, and K. Wells, "Development of a particle filter framework for respiratory motion correction in nuclear medicine imaging," in SPIE Medical Imaging Proceedings. San Diego, California, USA: SPIE, 2010.

[9] M. Alnowani, E. Lewis, M. Guy, and K. Wells, "A marker-less observation model for motion correction in nuclear medicine," in SPIE Medical Imaging Proceedings. San Diego, California, USA: SPIE, 2010.

[10] P. Bergstrom and G. Shechter, "Iterated closest points implemenatation," 2007, includes a version of $\mathrm{k}-\mathrm{d}$ tree.

[11] W. R. Crum, R. I. Scahill, and N. C. Fox, "Automated hippocampal segmentation by regional fluid registration of serial mri: Validation and application in alzheimer's disease," Neuroimage, vol. 13, no. 5, pp. 847855, 2001, times Cited: 63 .

[12] W. P. Segars, B. M. Tsui, D. S. Lalush, E. C. Frey, M. A. King, and D. Manocha, "Development and application of the new dynamic nurbs-based cardiac-torso (ncat) phantom," Journal of Nuclear Medicine, vol. 42, no. 5, pp. 7P-7P, 2001, suppl. S.

[13] M. J. Guy, G. D. Flux, P. Papavasileiou, M. A. Flower, and R. J. Ott, "RMDP: A dedicated package for i-131 spect quantification, registration and patient-specific dosimetry," Cancer Biotherapy and Radiopharmaceuticals, vol. 18, no. 1, pp. 61-69, 2003. 